\title{
Interobserver agreement in detection of "white globe appearance" and the ability of educational lectures to improve the diagnosis of gastric lesions
}

\author{
Hitoshi Omura ${ }^{1,4} \cdot$ Naohiro Yoshida $^{1}$ Tomoyuki Hayashi ${ }^{2} \cdot$ Kazuhiro Miwa $^{3}$ • \\ Hajime Takatori ${ }^{4}$ Hirokazu Tsuji ${ }^{5}$ Katsuhisa Inamura ${ }^{6} \cdot$ Yukihiro Shirota $^{7}$. \\ Hiroyuki Aoyagi ${ }^{8} \cdot$ Takaharu Masunaga $^{9}$ Kazuyoshi Katayanagi ${ }^{10}$. \\ Hiroshi Kurumaya $^{10} \cdot$ Shuichi Kaneko $^{4} \cdot$ Hisashi Doyama ${ }^{1}$
}

Received: 31 May 2016/ Accepted: 20 November 2016/Published online: 3 December 2016

(c) The International Gastric Cancer Association and The Japanese Gastric Cancer Association 2016

\begin{abstract}
Background White globe appearance (WGA) refers to a small white lesion of globular shape underneath cancerous gastric epithelium that can be clearly visualized by magnifying endoscopy with narrowband imaging (M-NBI). WGA has been reported to be a novel endoscopic marker that is highly specific in differentiating early gastric cancer (GC) from lowgrade adenoma, and has a significantly higher prevalence in early GCs than in noncancerous lesions. However, interobserver agreement in detecting WGA and whether training intervention can improve diagnostic accuracy are unknown. Methods Twenty sets of M-NBI images were examined by 16 endoscopists. The endoscopists attended a lecture about WGA, and examined the images again after the lecture. Interobserver agreement in detecting WGA in the second examination and increases in the proportion of correct
\end{abstract}

Electronic supplementary material The online version of this article (doi:10.1007/s10120-016-0676-3) contains supplementary material, which is available to authorized users.

Hisashi Doyama

doyama.134@ipch.jp

1 Department of Gastroenterology, Ishikawa Prefectural Central Hospital, 2-1 Kuratsukihigashi, Kanazawa, Ishikawa 920-8530, Japan

2 Department of Gastroenterology, Kanazawa Medical Center, Kanazawa, Ishikawa, Japan

3 Department of Gastroenterology, Japan Community Health care Organization Kanazawa Hospital, Kanazawa, Ishikawa, Japan

4 Department of Gastroenterology, Kanazawa University Hospital, Kanazawa, Ishikawa, Japan

5 Department of Gastroenterology, Kanazawa Municipal Hospital, Kanazawa, Ishikawa, Japan diagnoses and the degree of confidence of diagnoses of cancerous lesions were evaluated.

Results The kappa value for interobserver agreement in detecting WGA in the second examination was 0.735 . The proportion of correct diagnoses was significantly higher in the second examination compared with the first examination when WGA was present (95.5\% vs $55.4 \% ; P<0.001)$, but not when WGA was absent ( $61.6 \%$ vs $52.7 \% ; P=0.190)$. The proportion of correct diagnoses with a high degree of confidence was significantly higher in the second examination, both with WGA $(91.1 \%$ vs $29.5 \% ; P<0.001)$ and without WGA ( $36.6 \%$ vs $20.5 \%$; $P=0.031)$.

Conclusions The detection of WGA by endoscopists was highly reproducible. A brief educational lecture about WGA increased the proportion of correct diagnoses and the degree of confidence of diagnoses of GC with WGA.

Keywords Gastric cancer - Magnifying endoscopy · Narrowband imaging - White globe appearance

6 Department of Gastroenterology, Tonami General Hospital, Tonami, Toyama, Japan

7 Department of Gastroenterology, Saiseikai Kanazawa Hospital, Kanazawa, Ishikawa, Japan

8 Department of Gastroenterology, Fukui Prefectural Hospital, Fukui, Fukui, Japan

9 Department of Gastroenterology, Hokuriku Hospital, Kanazawa, Ishikawa, Japan

10 Department of Diagnostic Pathology, Ishikawa Prefectural Central Hospital, Kanazawa, Ishikawa, Japan 


\section{Introduction}

Magnifying endoscopy with narrowband imaging (M-NBI) is a powerful tool for the diagnosis of gastric mucosal lesions. The M-NBI system can visualize both the microvascular architecture and the microsurface structure in the superficial mucosa, with diagnoses based on these patterns [1-5]. M-NBI using the vessel-plus-surface classification system (VSCS) [6] was developed to diagnose gastric cancer (GC), with its diagnostic results matching those of histopathological diagnosis and delineation of lesion margins [7-9]. During screening endoscopy, M-NBI using the VSCS is useful for differentiating between early GC and other lesions, including gastritis. Furthermore, M-NBI using the VSCS can act as an "optical biopsy" if its diagnosis has a high degree of confidence when mucosal lesions that are reddened or the same color as the background mucosa are being assessed [9]. Especially, on the basis of the published data obtained with the use of the VSCS [3, 5-9], the Japanese Gastroenterological Association proposed that the Japan Gastroenterological Endoscopy Society, the Japanese Gastric Cancer Association, and the World Endoscopy Organization jointly devise a unified international algorithm for magnifying endoscopy diagnosis of early GC using an evidence-based approach [10].

Endoscopists still face challenges in using M-NBI and the VSCS in clinical practice. The diagnostic reproducibility of this system was found to be relatively unsatisfactory, with a mean kappa value for interobserver agreement of 0.50 [11]. Furthermore, the mean diagnostic interobserver kappa value for trainees who had been performing endoscopies for less than 1 year but had never used M-NBI was only 0.40 [12]. In addition, the degree of confidence within each M-NBI diagnostic category (e.g., cancerous or noncancerous lesions) was found to be wide-ranging, from low to high confidence. The proportion of correct diagnoses with a high degree of confidence was reported to be only $50 \%$ in patients with a pathological diagnosis of cancer by screening endoscopy using M-NBI [9]. In addition, a method of easing the learning curve for M-NBI procedures has not yet been established [9].

We recently described a novel endoscopic finding, "white globe appearance" (WGA), wherein a white globe is defined as a small (less than $1 \mathrm{~mm}$ ) white lesion of globular shape that is found underneath the gastric epithelium and can be clearly visualized by M-NBI [13]. Histopathologically, WGA was found to correspond to intraglandular necrotic debris within markedly dilated glands [13, 14]. Intraglandular necrotic debris was reported to be a possible histological marker for cancer [14]. A retrospective study showed that the presence of WGA had
$100 \%$ specificity in differentiating early GC from lowgrade adenoma [13]. Moreover, a prospective study showed that the prevalence of WGA was significantly higher in GCs than in noncancerous lesions, including gastritis (21.4\% vs 2.5\%) [15]. Taken together, these results suggest that the presence of WGA may have high specificity and a high positive predictive value in the diagnosis of GC, and that a positive WGA finding in a suspicious lesion on M-NBI would be an adjunct to the M-NBI diagnosis of possible GC. In these reports, the WGA's high validity in the diagnosis of GC was demonstrated. Additionally, the diagnostic criteria for WGA are simple and explicit, based only on its presence or absence, suggesting that endoscopists can easily learn to identify these lesions. To date, however, no study has investigated the agreement in detecting WGA or whether training in the identification of WGA can increase diagnostic accuracy. This study therefore aimed to evaluate the interobserver agreement in detecting WGA, and to determine whether education about WGA can increase the proportion of correct diagnoses and the degree of confidence of M-NBIguided diagnoses.

\section{Methods}

\section{Study design and participating endoscopists}

The trial included 16 endoscopists with various levels of experience from eight different institutions in Japan. The endoscopists provided information about the number of years of experience performing upper gastrointestinal tract endoscopy, the number of M-NBI procedures they had performed throughout their career, and whether they were board-certified by the Japan Gastroenterological Endoscopy Society. At the start of this study, WGA had never been reported in the literature or in conference presentations; thus, the participants were unfamiliar with WGA, although most were familiar with M-NBI.

All participants took an examination approximately 1 week before participating in a brief, 15-min educational lecture about WGA given by an expert endoscopist (H.D.) familiar with WGA. The lecture included information about the definition of WGA, its correspondence with pathological findings, and its specificity for GC. Participants took a second examination within 2 weeks after the lecture. In the examinations, the participants judged whether lesions were cancerous or noncancerous and their confidence level for the diagnosis in each of 20 lesions. Furthermore, in the second examination, they judged whether WGA was present or absent independently of their diagnoses of cancerous or noncancerous lesions. 
Interobserver agreement in detecting WGA was assessed in the second examination, as was the change in the proportion of correct diagnoses (e.g., GC or noncancerous lesions) from the first to the second examination and the confidence in assessing cancerous lesions in both examinations (Fig. 1).

This study on humans was performed in accordance with the declaration of Helsinki and was approved by the Institutional Review Board of Ishikawa Prefectural Central Hospital.

\section{Endoscopic images}

Before this study, the expert endoscopist (H.D.) chose 20 sets of M-NBI images containing cancerous and noncancerous lesions from the endoscopy databases at Ishikawa Prefectural Central Hospital; all patients had also undergone endoscopic submucosal dissection or biopsy. All cancerous lesions had been diagnosed by pathological examination of a specimen obtained by endoscopic submucosal dissection. All noncancerous lesions had been diagnosed by pathological examination of a specimen obtained biopsy. For the purpose of maintaining high accuracy of pathological diagnosis, noncancerous lesions were biopsied at least twice at another time.

The images included those of 14 GCs (including nine well-differentiated adenocarcinomas, four moderately differentiated adenocarcinomas, and one undifferentiated adenocarcinoma) and six noncancerous lesions from patients with chronic gastritis. The expert endoscopist (H.D.) determined that WGA was present in seven GCs and was absent in the other seven GCs and the six noncancerous lesions. The characteristics and endoscopic features of the cancerous and noncancerous lesions are presented in the electronic supplementary material.

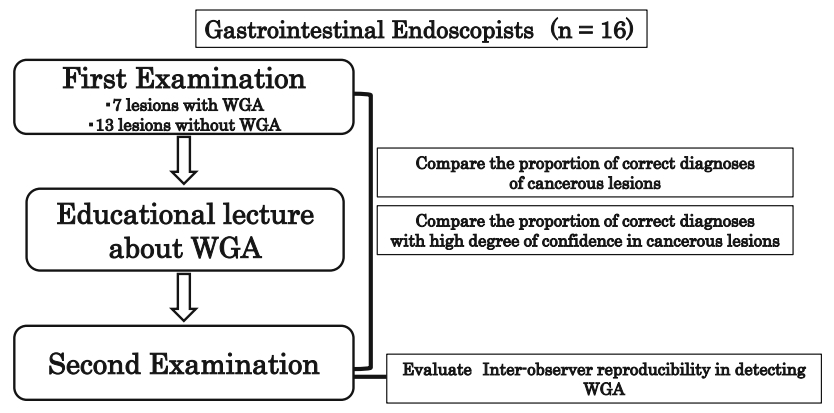

Fig. 1 Protocol design of the study. Sixteen endoscopists participated in this study. They took the first examination before receiving the lecture and the second examination after the lecture. Interobserver agreement in detecting white globe appearance (WGA) in the second examination was assessed. Additionally, the change in the proportion of correct diagnoses of cancerous lesions and the change in the proportion of correct diagnoses with a high degree of confidence of cancerous lesions were evaluated
Pathological diagnosis was based on the revised Vienna classification [16]: C4 (mucosal high-grade neoplasia) and C5 (submucosal invasion by carcinoma) were diagnosed as GC, with all other categories diagnosed as noncancerous lesions. GCs were histologically typed according to the Japanese classification of gastric carcinoma by two expert pathologists (H.K. and K.K.), with the results of all lesions double-checked [16, 17].

All endoscopists were shown three to four M-NBI images per lesion, including images taken at maximal magnification. The endoscopist were not shown nonmagnified narrowband imaging or white-light endoscopy images to avoid any confusion. If white-light endoscopy and chromoendoscopy images were included in the examination, participants' diagnoses might be affected in some degree by interpretation of these, so to evaluate accurately the value of WGA visualized by M-NBI, such images were not included in the examination. Each lesion was numbered and the sets of images were randomized, with their number displayed in the second examination to avoid bias.

\section{Endoscopic system and settings}

M-NBI was performed with a magnifying endoscope (GIFH260Z, Olympus, Tokyo, Japan) equipped with a soft black hood (MAJ-1990 for GIF-H260Z, Olympus), a video processor (EVIS LUCERA CV-260SL, Olympus), and a light source (CV-260SL, Olympus). The video processor settings for M-NBI included structure enhancement set to B-mode level 8 and color mode set to level 1 .

\section{Definition of endoscopic findings of WGA}

WGA was defined as a small white lesion of globular shape. Two characteristics were useful in identifying WGA: (1) intensification of whiteness from the margins to the center, reflecting globular shape; and (2) the presence of microvessels overlying the WGA, because of its position underneath the gastric epithelium and subepithelial microvessels. The presence of a WGA was defined as its occurrence in at least one image from each set of images; absence was defined as the absence in all images of that set (Fig. 2).

\section{Diagnostic criteria for M-NBI}

According to the VSCS diagnostic criteria, lesions were considered cancerous on M-NBI (Fig. 2) if they showed an irregular microvascular and/or microsurface pattern with a demarcation line. The absence of both was defined as an endoscopic diagnosis of noncancerous lesions. Additionally, in the second examination, a positive WGA finding could be an adjunct to the diagnosis of possible cancer. As 
WGA shows high specificity for GC, it may be a diagnostic marker for GC; however, WGA had low sensitivity, indicating that the absence of WGA is not diagnostic of noncancerous lesions.

\section{Degree of confidence of endoscopic diagnoses}

The grading system indicating the degree of M-NBIguided diagnostic confidence has been described [9]. Specifically, grades 1 and 2 indicate noncancerous lesions with a high and low degree of confidence respectively, grade 3 is indeterminate, and grades 4 and 5 indicate GC with a low and high degree of confidence respectively. Grades 1-3 were classified as noncancerous lesions, and grades 4 and 5 were classified as GC, with grades 1 and 5 classified as prediction with high confidence and grades 2-4 classified as prediction with low confidence.

\section{Outcome measures}

The primary outcome was to investigate interobserver agreement in detecting WGA in the second examination. Secondary outcome measures included (1) the proportion of correct diagnoses in detecting the presence or absence of WGA in the second examination, when compared with the expert; (2) increase in the proportion of correctly diagnosed cancerous lesions with and without WGA; (3) increase in the proportion of correct diagnoses with a high degree of confidence of cancerous lesions with and without WGA. These outcomes were compared in subgroups of endoscopists, including those who were and were not boardcertified by the Japan Gastroenterological Endoscopy Society, and on the basis of their relative experience with upper gastrointestinal tract endoscopy and the M-NBI procedure.

\section{Statistical analysis}

The means were compared by paired $t$ tests for two paired groups. All $P$ values calculated in this analysis were twosided, and $P<0.05$ was considered statistically significant. The kappa value was calculated to evaluate the agreement in WGA identification among the participants. The accuracy of diagnosis was calculated by standard formulas. All analyses were performed with $\mathrm{R}$ version 3.0.2 (Comprehensive R Archive Network, http://cran.r-project.org) and JMP 11 (SAS Institute, Cary, NC, USA).
Fig. 2 Example of the sets of magnifying endoscopy with narrowband imaging images used for the examinations. Endoscopic image of white globe appearance (WGA) visualized as a small, white lesion with globular shape by magnifying endoscopy with narrowband imaging with maximal magnification. White circles show WGA. The following two characteristics are useful for identifying the WGA: (1) the whiteness intensifies from the margins to the center, reflecting its globular shape, and (2) microvessels overly the WGA, because of its position underneath the gastric epithelium and the subepithelial microvessels. This is a set of the cancerous lesion with a demarcation line (yellow arrow) and irregular microvascular and microsurface patterns. This lesion was endoscopically resected and diagnosed as gastric cancer
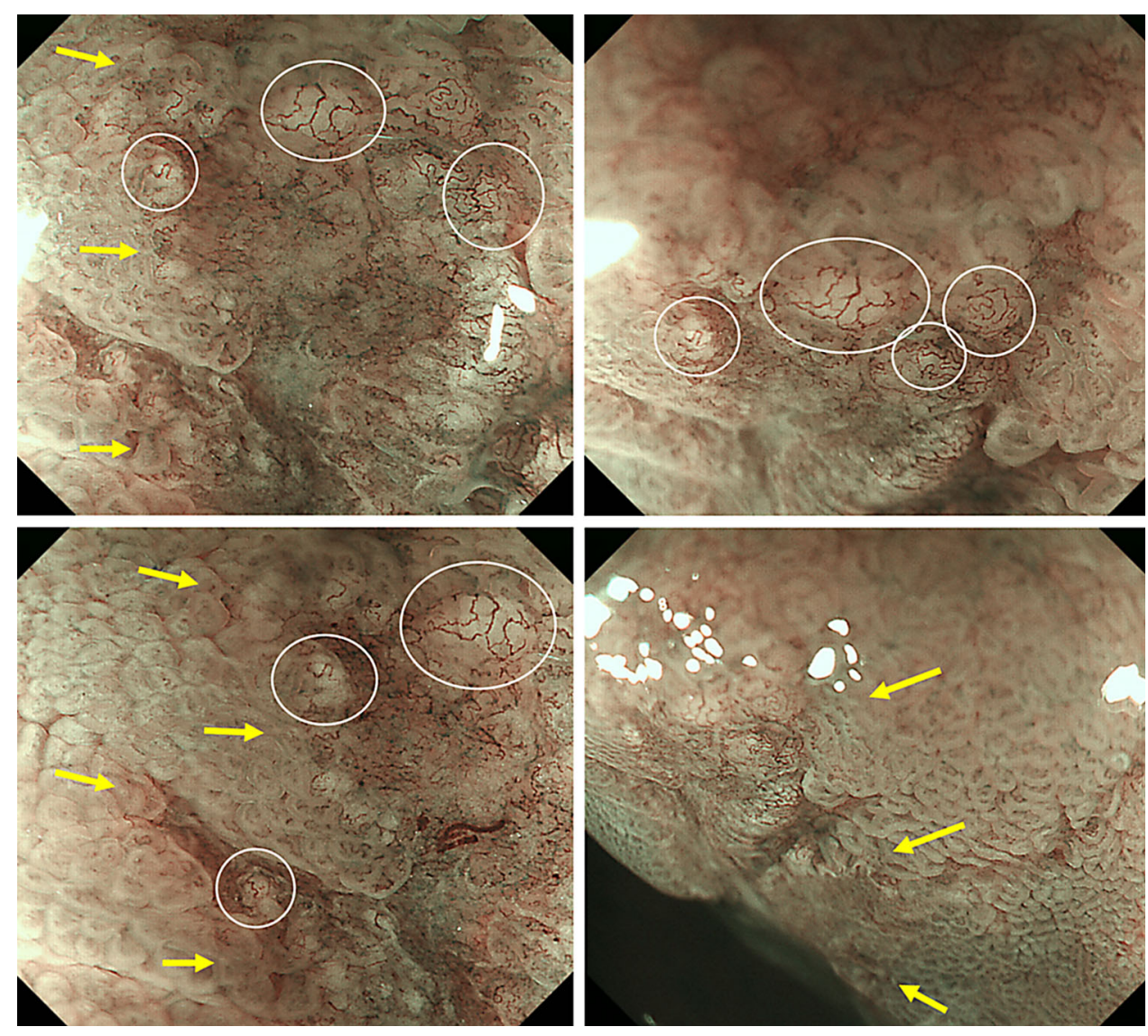
Table 1 Characteristics of the participating endoscopists and interobserver agreement for detecting white globe appearance

\begin{tabular}{lcc}
\hline & Number & Kappa \\
\hline $\begin{array}{l}\text { Board-certified by JGES } \\
\text { Yes }\end{array}$ & 11 & \\
No & 5 & 0.709 \\
Years of experience performing & 0.829 \\
upper gastrointestinal tract endoscopy & \\
$\geq 13$ & 8 & \\
$<13$ & 8 & 0.744 \\
Number of M-NBI procedures performed & 0.753 \\
$\geq 100$ & 9 & \\
$<100$ & 7 & 0.699 \\
All participants & 16 & 0.800 \\
\hline
\end{tabular}

JGES Japan Gastroenterological Endoscopy Society, M-NBI magnifying endoscopy with narrowband imaging

\section{Results}

Sixteen endoscopists attended the lecture, completed the examinations, and were eligible for analysis in this study. The characteristics of these participants are summarized in Table 1.

In the second examination, the overall kappa value for interobserver agreement in detecting WGA in all 20 lesions was 0.735 . In subgroups of these participants (i.e., those who were or were not board-certified by the Japan Gastroenterological Endoscopy Society and those subgrouped by years of experience of performing upper gastrointestinal tract endoscopy and by the number of M-NBI procedures), the kappa values ranged from 0.699 to 0.829 , indicating substantial or almost perfect agreement (Table 1).

The diagnostic accuracy, sensitivity, and specificity for all 20 lesions were $60.0 \%, 54.0 \%$, and $74.0 \%$ in the first examination, and $67.5 \%, 78.6 \%$, and $41.7 \%$ in the second examination for all participants. The accuracy in detecting WGA when WGA was present in the lesions was 84.8\% (95/ 112 ), whereas the accuracy in not detecting WGA when WGA was absent from the lesions was $96.2 \%$ (200/208).

The average proportions of correct diagnoses of cancerous lesions with WGA were $55.4 \%$ in the first examination and $95.5 \%$ in the second examination, a differential of 40.1 percentage points $(P<0.001)$. In comparison, the average proportions of correct diagnoses of cancerous lesions without WGA were $52.7 \%$ in the first examination and $61.6 \%$ in the second examination, a differential of 8.9 percentage points $(P=0.190)$. The differential was significantly higher in the former than in the latter group of lesions (40.1 percentage points vs 8.9 percentage points, $P=0.002$ ) (Fig. 3). The proportion of correct diagnoses of cancerous lesions when WGA was present increased significantly from the first to the second examination in all subgroups of participants. In the

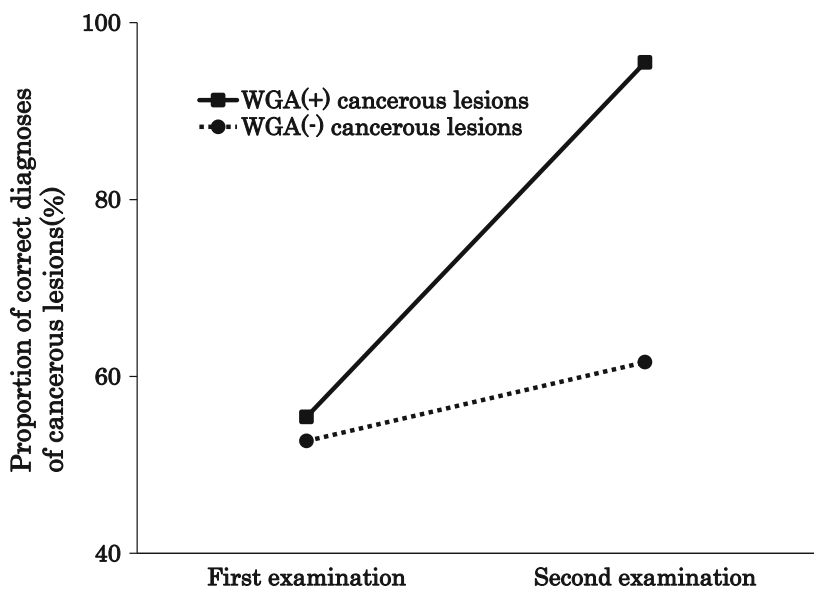

Fig. 3 The changes in the proportion of correct diagnoses of cancerous lesions in the first and second examinations. A significant increase in the proportion of correct diagnoses was observed for cancerous lesions with white globe appearance (WGA) $(55.4 \%$ to 95.5\%; differential of 40.1 percentage points, $P<0.001)$ but there was no significant difference in the proportion of correct diagnoses of cancerous lesions without WGA $(52.7 \%$ to $61.6 \%$; differential of 8.9 percentage points, $P=0.190)$. The increase in the rate was significantly higher in lesions with WGA than in lesions without WGA $(P=0.002)$

absence of WGA, however, the proportion of correct diagnoses of cancerous lesions increased significantly in nonboard-certified endoscopists, from $57.1 \%$ in the first examination to $88.6 \%$ in the second examination $(P=0.040)$, but did not increase significantly in any other subgroup (Table 2).

Participants showed a significant increase in correct diagnoses with a high degree of confidence (i.e., grade 5) in the second examination compared with the first examination when they evaluated cancerous lesions with WGA $(91.1 \%$ vs $29.5 \%, P<0.001)$ and without WGA $(36.6 \%$ vs $20.5 \%, P=0.031$ ), although the differential was significantly higher in the former group of lesions than in the latter group (61.6 percentage points vs 16.1 percentage points, $P<0.001$ ) (Fig. 4). All subgroups of participants showed a significant increase in correct diagnoses with a high degree of confidence of cancerous lesions with WGA from the first to the second examination. Only the subgroup of participants with less than 13 years' experience with upper gastrointestinal tract endoscopy showed a significant increase in the proportion of correct diagnoses with a high degree of confidence of cancerous lesions without WGA from the first to the second examination (Table 3).

\section{Discussion}

This study showed that all endoscopists, regardless of experience and board certification, were able to detect WGA. A recent study reported a high degree of agreement 
Table 2 Proportion of correct diagnoses of cancerous lesions in the first and second examinations in subgroups of endoscopists

\begin{tabular}{|c|c|c|c|}
\hline & 1st examination & 2nd examination & $P^{\mathrm{a}}$ \\
\hline \multicolumn{4}{|c|}{ Cancerous lesions with WGA $(n=7)$} \\
\hline \multicolumn{4}{|c|}{ Board-certified by the JGES } \\
\hline Yes $(n=11)$ & 0.558 & 0.935 & $<0.001$ \\
\hline No $(n=5)$ & 0.543 & 1 & 0.005 \\
\hline \multicolumn{4}{|c|}{ Years of experience performing upper gastrointestinal tract endoscopy } \\
\hline$\geq 13(n=8)$ & 0.607 & 0.929 & 0.007 \\
\hline$<13(n=8)$ & 0.500 & 0.982 & $<0.001$ \\
\hline \multicolumn{4}{|c|}{ Number of M-NBI procedures } \\
\hline$\geq 100(n=9)$ & 0.571 & 0.937 & 0.001 \\
\hline$<100(n=7)$ & 0.530 & 0.980 & 0.002 \\
\hline \multicolumn{4}{|c|}{ Cancerous lesions without WGA $(n=7)$} \\
\hline \multicolumn{4}{|c|}{ Board-certified by the JGES } \\
\hline Yes $(n=11)$ & 0.506 & 0.494 & 0.839 \\
\hline No $(n=5)$ & 0.571 & 0.886 & 0.040 \\
\hline \multicolumn{4}{|c|}{ Years of experience performing upper gastrointestinal tract endoscopy } \\
\hline$\geq 13(n=8)$ & 0.482 & 0.518 & 0.598 \\
\hline$<13(n=8)$ & 0.571 & 0.714 & 0.252 \\
\hline \multicolumn{4}{|c|}{ Number of M-NBI procedures } \\
\hline$\geq 100(n=9)$ & 0.524 & 0.651 & 0.121 \\
\hline$<100(n=7)$ & 0.531 & 0.571 & 0.745 \\
\hline
\end{tabular}

JGES Japan Gastroenterological Endoscopy Society, M-NBI magnifying endoscopy with narrowband imaging, $W G A$ white globe appearance

${ }^{\text {a }}$ Paired $t$ test

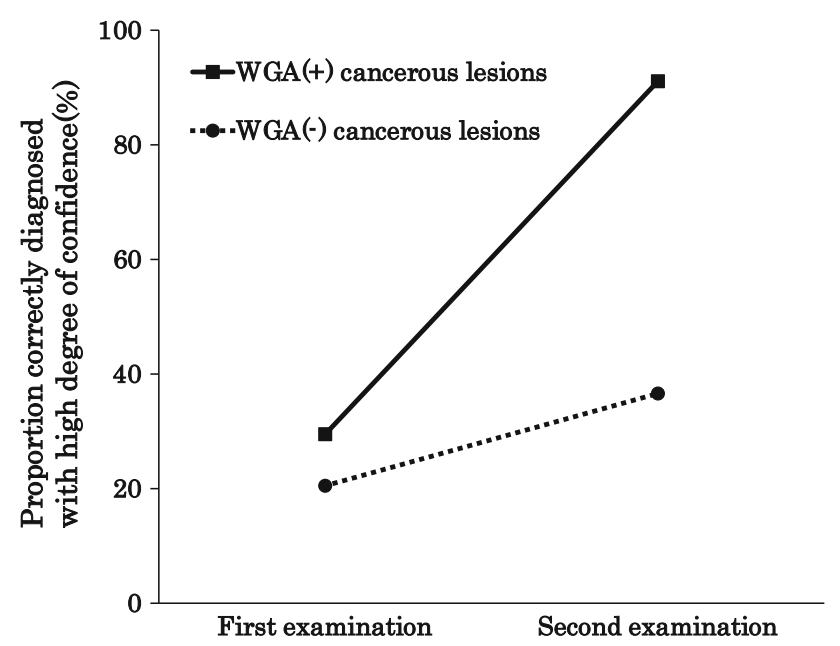

Fig. 4 The average proportion of correct diagnoses with a high degree of confidence of cancerous lesions. A significant increase in the proportion of correct diagnoses with a high degree of confidence was observed both for cancerous lesions with white globe appearance (WGA) $(29.5 \%$ to $91.1 \%$; differential of 61.6 percentage points, $P<0.001)$ and for cancerous lesions without WGA $(20.5 \%$ to $36.6 \%$; differential of 16.1 percentage points, $P=0.031$ ). The increase in the rate was significantly higher in lesions with WGA compared with lesions without WGA $(P<0.001)$ in WGA identification on routine M-NBI endoscopy between eight trainees and one expert in a single institution (kappa value 0.902) [15]. This trial evaluated interobserver agreement in detecting WGA following initial education about WGA. Despite these endoscopists practicing at various institutions and having a wide range of experience, interobserver agreement was substantial (kappa value 0.735). In addition, the accuracy in determining the presence or absence of WGA, relative to that of the expert endoscopist, was high. With regard to cancerous lesions with WGA, it is quite possible that WGA enhance the reproducibility in diagnosing GC.

The overall proportion of correct diagnoses of cancerous lesions of our 16 participants in the first examination was $54.0 \%$. This poor performance might have been due to the participants' inexperience with M-NBI procedures. With the regard of the number of M-NBI procedures in their whole career, 13 endoscopists had performed fewer than 500 procedures, and 7 of them had performed fewer than 100 procedures, and so they would not have great experience of M-NBI. However, in a similar study, Mabe et al.[18] reported the proportion of correct diagnoses of malignant lesions as $47.0 \%$ for 64 endoscopists in an 
Table 3 Proportion of correct diagnoses with a high degree of confidence of cancerous lesions in the first and second examinations by subgroups of participants

\begin{tabular}{|c|c|c|c|}
\hline & 1st examination & 2nd examination & $P^{\mathrm{a}}$ \\
\hline \multicolumn{4}{|c|}{$\begin{array}{l}\text { Cancerous lesions with WGA } \\
(n=7)\end{array}$} \\
\hline \multicolumn{4}{|c|}{ Board-certified by the JGES } \\
\hline Yes $(n=11)$ & 0.273 & 0.883 & $<0.001$ \\
\hline No $(n=5)$ & 0.343 & 0.971 & $<0.001$ \\
\hline \multicolumn{4}{|c|}{ Years of experience performing upper gastrointestinal tract endoscopy } \\
\hline$\geq 13(n=8)$ & 0.286 & 0.857 & $<0.001$ \\
\hline$<13(n=8)$ & 0.304 & 0.964 & $<0.001$ \\
\hline \multicolumn{4}{|c|}{ Number of M-NBI procedures } \\
\hline$\geq 100(n=9)$ & 0.238 & 0.889 & $<0.001$ \\
\hline$<100(n=7)$ & 0.367 & 0.939 & $<0.001$ \\
\hline \multicolumn{4}{|c|}{$\begin{array}{l}\text { Cancerous lesions without WGA } \\
(n=7)\end{array}$} \\
\hline \multicolumn{4}{|c|}{ Board-certified by the JGES } \\
\hline Yes $(n=11)$ & 0.156 & 0.208 & 0.267 \\
\hline No $(n=5)$ & 0.314 & 0.714 & 0.059 \\
\hline \multicolumn{4}{|c|}{ Years of experience performing upper gastrointestinal tract endoscopy } \\
\hline$\geq 13(n=8)$ & 0.161 & 0.214 & 0.402 \\
\hline$<13(n=8)$ & 0.250 & 0.518 & 0.049 \\
\hline \multicolumn{4}{|c|}{ Number of M-NBI procedures } \\
\hline$\geq 100(n=9)$ & 0.190 & 0.286 & 0.242 \\
\hline$<100(n=7)$ & 0.224 & 0.469 & 0.087 \\
\hline
\end{tabular}

examination involving $20 \mathrm{M}-\mathrm{NBI}$ images. Our participants were considered to have the same level of diagnostic accuracy as those in that study.

The results of this study also demonstrated that a brief educational lecture about WGA significantly increased the proportion of correct diagnoses of early GCs with WGA, to $95.5 \%$ in the second examination. This increase was observed in all subgroups, regardless of the experience with upper gastrointestinal tract endoscopy and M-NBI procedures, suggesting that identification of WGA can be learned easily and that it is an adjunct marker for early GC. The criterion we used, whether WGA was present or absent, is simple, explicit, and easily comprehensible and may increase the proportion of correct diagnoses of cancerous lesions with WGA.

This study also showed that learning about WGA could increase the degree of diagnostic confidence. The proportion of cancerous lesions with WGA correctly diagnosed with a high degree of confidence increased dramatically, from $29.5 \%$ before the lecture to $91.1 \%$ after the lecture (an increase of 61.6 percentage points), with strong increases in the degree of confidence observed in all subgroups of endoscopists. A positive WGA finding in a cancerous lesion would be a strong adjunct to M-NBI diagnosis with the VSCS.

The clinical impact of WGA on the M-NBI diagnosis of GC is largely dependent on the frequency of WGA among GCs. Prospective and retrospective studies found that WGA was present in $21.4 \%$ and $21.5 \%$, respectively, of differentiating GCs $[13,15]$. However, GCs with WGA were significantly smaller than GCs without WGA. Moreover, we found that the prevalence of WGA in GCs $10 \mathrm{~mm}$ or smaller in diameter was $42.1 \%$ (unpublished data). In practice, identifying cancer-specific characteristics is relatively difficult, especially for small GCs, with the sensitivities for minute ( $5 \mathrm{~mm}$ or smaller) and small (10 mm or smaller) GCs being 88.3\% [19] and $60.0 \%$ [3], respectively. The presence of WGA will make a strong contribution to the correct diagnosis of small gastric lesions, especially those $10 \mathrm{~mm}$ or smaller.

The incidence of WGA in noncancerous lesions was reported to be only $2.5 \%$, being observed in noncancerous lesions suspected of being GCs; these included a benign open ulcer, a patient with gastritis, and a low-grade adenoma with an ulcer scar [15]. We were unable to prepare noncancerous lesions with WGA and therefore could not 
compare the diagnostic performance of this system in noncancerous lesions with and without WGA. Nevertheless $3.1 \%(3 / 96)$ of lesions were found to be falsely positive for WGA, resulting in their misdiagnosis. The diagnosis of GC by M-NBI should be based not only on the presence or absence of WGA but also on the VSCS.

This study had several limitations. First, it was not an in vivo study. Rather, this study evaluated high-quality still images of maximal magnification taken by one expert endoscopist. In addition, the recording technique used by the participants was not evaluated. In practice, sharp still M-NBI images are required for an accurate diagnosis [20]. Second, no formal sample size was estimated because this study was regarded as exploratory. Third, there was a selection bias of the M-NBI images for the examination. These images were chosen such that the mean diagnostic accuracy would be the same in images of GC with WGA, GC without WGA, and noncancerous lesions. For the purpose of this study, the expert endoscopist used the results of previous training examinations performed before the first identification of WGA. Therefore, these prepared images did not mimic a real-world endoscopic diagnosis. These limitations need to be taken into account when readers are considering this study and its contributions.

In conclusion, the endoscopic detection of WGA is highly reproducible, and a brief educational lecture about WGA can increase both the proportion of correct diagnoses and the degree of confidence of M-NBI-guided diagnoses of GC with WGA. WGA may serve as a reliable and easily learnable marker of early GC, irrespective of endoscopists' experience.

Acknowledgements The authors thank the participating endoscopists: Kiichiro Kaji (Kanazawa Medical Center), Fumiyo Iida (Japan Community Health care Organization Kanazawa Hospital), Yoshimoto Nomura (Kanazawa Municipal Hospital), Akihiko Kida and Shogo Matsuda (Saiseikai Kanazawa Hospital), Yoshihide Naitou (Fukui Prefectural Hospital), and Toshiki Sakashita (Hokuriku Hospital).

\section{Compliance with ethical standards}

Conflict of interest The authors declare that they have no conflict of interest.

Human rights statement and informed consent All procedures followed were in accordance with the ethical standards of the responsible committee on human experimentation (institutional and national) and with the Helsinki Declaration of 1964 and later versions. Informed consent or substitute for it was obtained from all patients for their being included in the study.

\section{References}

1. Muto M, Katada C, Sano Y, Yoshida S. Narrow band imaging: a new diagnostic approach to visualize angiogenesis in superficial neoplasia. Clin Gastroenterol Hepatol. 2005;3:S16-20.
2. Yao K, Oishi T, Matsui T, Yao T, Iwashita A. Novel magnified endoscopic findings of microvascular architecture in intramucosal gastric cancer. Gastrointest Endosc. 2002;56:279-84.

3. Ezoe Y, Muto M, Uedo N, Doyama H, Yao K, Oda I, et al. Magnifying narrowband imaging is more accurate than conventional white-light imaging in diagnosis of gastric mucosal cancer. Gastroenterology. 2011;141:2017-25. doi:10.1053/j.gastro.2011. 08.007.

4. Kaise M, Kato M, Urashima M, Arai Y, Kaneyama H, Kanzazawa $\mathrm{Y}$, et al. Magnifying endoscopy combined with narrowband imaging for differential diagnosis of superficial depressed gastric lesions. Endoscopy. 2009;41:310-5. doi:10.1055/s-00281119639.

5. Yamada S, Doyama H, Yao K, Uedo N, Ezoe Y, Oda I, et al. An efficient diagnostic strategy for small, depressed early gastric cancer with magnifying narrow-band imaging: a post-hoc analysis of a prospective randomized controlled trial. Gastrointest Endosc. 2014;79:55-63. doi:10.1016/j.gie.2013.07.008.

6. Yao K, Anagnostopoulos GK, Ragunath K. Magnifying endoscopy for diagnosing and delineating early gastric cancer. Endoscopy. 2009;41:462-7. doi:10.1055/s-0029-1214594.

7. Nagahama T, Yao K, Maki S, Yasaka M, Takaki Y, Matsui T, et al. Usefulness of magnifying endoscopy with narrow-band imaging for determining the horizontal extent of early gastric cancer when there is an unclear margin by chromoendoscopy (with video). Gastrointest Endosc. 2011;74:1259-67. doi:10. 1007/s10120-012-0160-7.

8. Miwa K, Doyama H, Ito R, Nakanishi H, Hirano K, Inagaki S, et al. Can magnifying endoscopy with narrow band imaging be useful for low grade adenomas in preoperative biopsy specimens? Gastric Cancer. 2012;15:170-8. doi:10.1007/s10120-011-0093-6.

9. Yao K, Doyama H, Gotoda T, Ishikawa H, Nagahama T, Yokoi $\mathrm{C}$, et al. Diagnostic performance and limitations of magnifying narrow-band imaging in screening endoscopy of early gastric cancer: a prospective multicenter feasibility study. Gastric Cancer. 2014;17:669-79. doi:10.1007/s10120-013-0332-0.

10. Muto M, Yao K, Kaise M, Kato M, Uedo N, Yagi K, et al. Magnifying endoscopy simple diagnostic algorithm for early gastric cancer (MESDA-G). Dig Endosc. 2016;28:379-93. doi:10.1111/den. 12638 .

11. Sumie H, Sumie S, Nakahara K, Watanabe Y, Matsuo K, Mukasa $\mathrm{M}$, et al. Usefulness of magnifying endoscopy with narrow-band imaging for diagnosis of depressed gastric lesions. Mol Clin Oncol. 2014;2:129-33.

12. Yoo CH, Park MI, Park SJ, Moon W, Kim HH, Song JY, et al. Observer variability in gastric neoplasm assessment using the vessel plus surface classification for magnifying endoscopy with narrow band imaging. Clin Endosc. 2014;47:74-8. doi:10.5946/ ce.2014.47.1.74.

13. Doyama H, Yoshida N, Tsuyama S, Ota R, Takeda Y, Nakanishi $\mathrm{H}$, et al. The "white globe appearance" (WGA): a novel marker for a correct diagnosis of early gastric cancer by magnifying endoscopy with narrow-band imaging (M-NBI). Endosc Int Open. 2015;3:E120-4. doi:10.1055/s-0034-1391026.

14. Watanabe Y, Shimizu M, Itoh T, Nagashima K. Intraglandular necrotic debris in gastric biopsy and surgical specimens. Ann Diagn Pathol. 2001;5:141-7.

15. Yoshida N, Doyama H, Nakanishi H, Tsuji K, Tominaga K, Matsunaga $\mathrm{K}$, et al. White globe appearance is a novel specific endoscopic marker for gastric cancer: a prospective study. Dig Endosc. 2015;28:59-66. doi:10.1111/den.12519.

16. Dixon MF. Gastrointestinal epithelial neoplasia: Vienna revisited. Gut. 2002;51:130-1.

17. Japanese Gastric Cancer Association. Japanese classification of gastric carcinoma: 3rd English edition. Gastric Cancer. 2011;14:101-12. 
18. Mabe K, Yao K, Nojima M, Tanuma T, Kato M. An educational intervention to improve the endoscopist's ability to correctly diagnose small gastric lesions using magnifying endoscopy with narrow-band imaging. Ann Gastroenterol. 2014;27:149-55.

19. Fujiwara S, Yao K, Nagahama T, Uchita K, Kanemitsu T, Tsurumi K, et al. Can we accurately diagnose minute gastric cancers $(\leq 5 \mathrm{~mm})$ ? Chromoendoscopy (CE) vs magnifying endoscopy with narrow band imaging (M-NBI). Gastric Cancer. 2015;18:590-6. doi:10.1007/s10120-014-0399-2.

20. Hayashi T, Doyama H, Shirota Y, Tsuji H, Marukawa Y, Ohta H, et al. Assessment of still and moving images in the diagnosis of gastric lesions using magnifying narrow-band imaging in a prospective multicenter trial. PLoS ONE. 2014;9:e100857. doi:10.1371/journal.pone.0100857. 